\title{
ROLE OF THE ANTIFERROMAGNETIC BULK SPINS IN EXCHANGE BIAS
}

\author{
Ivan K. Schuller ${ }^{a}$, Rafael Morales ${ }^{b, *}$, Xavier Batlle ${ }^{c}$, Ulrich Nowak ${ }^{\mathrm{d}}$, Gernot \\ Güntherodt $\mathrm{e}^{\mathrm{e}}$. \\ ${ }^{a}$ Center for Advanced Nanoscience and Physics Department, University of California San Diego, \\ La Jolla, CA. 92093, USA. \\ ${ }^{\mathrm{b}}$ Department of Chemical-Physics \& BCMaterials, University of the Basque Country UPV/EHU, \\ and IKERBASQUE, Basque Foundation for Science, 48011 Bilbao, Spain. \\ ${ }^{\mathrm{c}}$ Departament Física Fonamental and Institut de Nanociència i Nanotecnologia, Universitat de \\ Barcelona, c/ Martí i Franqués s/n, 08028 Barcelona, Catalonia, Spain. \\ ${ }^{\mathrm{d}}$ Department of Physics, University of Konstanz, 78464 Konstanz, Germany. \\ ' Physics Institute (IIA), RWTH Aachen University, Campus RWTH-Melaten, 52074 Aachen, \\ Germany. \\ * Corresponding author. \\ E-mail: rafael.morales@ehu.es (R. Morales)
}




\begin{abstract}
This "Critical Focused Issue" presents a brief review of experiments and models which describe the origin of Exchange Bias in epitaxial or textured ferromagnetic/antiferromagnetic bilayers. Evidence is presented which clearly indicates that inner, uncompensated, pinned moments in the bulk of the antiferromagnet (AFM) play a very important role in setting the magnitude of the Exchange Bias. A critical evaluation of the extensive literature in the field indicates that it is useful to think of this bulk, pinned uncompensated moments as a new type of a ferromagnet which has a low total moment, an ordering temperature given by the AFM Néel temperature, with parallel aligned moments randomly distributed on the regular AFM lattice.
\end{abstract}

Keywords (maximum of 6 keywords): exchange bias; antiferromagnet; ferromagnet; exchange coupling; bulk antiferromagnetic spins/moments. 


\section{INTRODUCTION}

Exchange Bias (EB) is characterized by the shift of the magnetic hysteresis loop along the field axis, generally observed in Antiferromagnetic (AFM)/Ferromagnetic (FM) bilayered hybrids [1]. This interesting, basic research effect is also the basis for many applications in the spintronics area such as magnetic data storage and sensor devices. The essential characteristics which determine the properties of an exchange biased system are: the magnitude of the shift, its sign, asymmetry of the hysteresis loop, blocking temperature (above which the EB disappears), training effect and time dependence. Although much work has been dedicated to understand the phenomenology of EB [2], each one of these important characteristics presents interesting puzzles, which give complementary clues regarding the essential physics of the effect. EB is generally considered to be a consequence of the interfacial interaction between the FM and AFM constituents [3]. This is attributed to the pinned, uncompensated magnetic moments [1,46] at the interface originating from the AFM.

Originally it was postulated that only the AFM interface controls the EB, i.e. EB is a purely interfacial phenomenon in which the role of the AFM bulk is restricted to pinning the interfacial magnetic moments. However, the interface is always coupled to the AFM bulk. Therefore the AFM bulk may affect the precise magnetic state of the interface with the consequent effect on the exchange bias. There is by now much compelling evidence that the bulk magnetic state of the AFM may affect the exchange bias, which implies that EB is not a purely interfacial phenomenon. Although its ultimate origin is the exchange interaction at the AFM-FM interface, the pinned, uncompensated spin distribution at the interface might be determined by the AFM bulk. In this "Critical Focused Issue" we highlight the role and microscopic origin of the pinned, uncompensated moments (PUM) present in the bulk of the AFM. More specifically, we emphasize the important experiments, which provide clues regarding the microscopic mechanism that governs Exchange Bias. We conclude by describing potential new directions in which this field can move and connected open questions. 
EB is initiated by cooling the FM/AFM bilayer in an externally applied magnetic field below the AFM Néel temperature. The exchange coupling between the FM and the AFM, which shifts the hysteresis loop along the field axis, is determined by an effective "exchange field" or by a "unidirectional" anisotropy energy. The AFM crystallinity, its morphology (e.g., grains) and intrinsic anisotropy are crucial parameters which determine the magnitude of the EB. In general, two types of exchange-biased systems, which show distinctly different behavior, can be distinguished. Type 1 are highly textured or epitaxial systems such as $\mathrm{FeF}_{2}$ or $\mathrm{CoO}$. On the other hand, type 2 are usually small-grained, polycrystalline systems, such as the classic archetypes IrMn or FeMn. It should be noted that the anisotropy energy, the central quantity determining the EB magnitude, depends on both the effective anisotropy constant as well as the crystal volume. Consequently, some polycrystalline AFMs may behave as either type 1 or type 2 depending on the crystallite size, the inter-crystallite magnetic coupling, which may lead to a larger effective particle volume, and the microstructure (e.g., growth mode), which may yield an effective increase of the anisotropy constant [7,8]. It is also important that the properties of the exchange bias bilayers are not only determined by the AFM's physical structure, but also its magnetic structure. Even if the crystallographic orientation of the AFM-FM interface is well defined, the spin orientation may become very complicated since in some cases equivalent crystallographic directions may not be magnetically equivalent. For instance, $\mathrm{NiO}$ is a classic example in which the (111) crystallographic plane has 4 structurally equivalent, but magnetically inequivalent directions.

Type 1 systems have in general a large exchange bias, the blocking temperature coincides with the AFM Néel temperature, and training and time dependences are practically absent. In type 2 systems the blocking temperature can be considerably reduced compared to the Néel temperature, and they may exhibit large training as well as time dependent effects. The change from negative (NEB) to positive (PEB) exchange bias shift can be present in both types of systems if the exchange coupling at the interface is antiferromagnetic and the surface layer of the AFM couples to the increasing external cooling field $[9,10]$. Like with many other situations in physics, there is no clear demarcation between type 1 and type 2 systems; these are just two extreme cases. For 
instance, there may be situations in which the blocking temperature coincides with the AFM Néel temperature but the systems exhibit large training effects [11-13]. This may occur even within the same combination of materials, since sometimes there are large structural differences within the same system. A classic example is $\mathrm{Co} / \mathrm{CoO}$ where the $\mathrm{CoO}$ may be polycrystalline, textured, or epitaxial depending on the specific preparation method.

There are a number of additional extrinsic experimental complications, which may cause confusion. Sometimes the exchange bias is much smaller than the coercivity, the hysteresis loops are sheared and/or there are large contributions from other (presumably irrelevant) parts of the sample such as substrates. In either case, small shifts along the field axis may be caused by artifacts such as vertical loop shifts and may complicate the identification of the EB. Other important issues which are not discussed here include intrinsic and extrinsic effects such as interfacial roughness, interdiffusion, variation in thickness and reduced magnetization and/or formation of interfacial compounds at interfaces and surfaces. Of course, in order to avoid erroneous conclusions the physical and chemical properties of these systems must be thoroughly characterized quantitatively using a comprehensive battery of tests.

\section{ISSUES}

In spite of all the above-mentioned complications, it seems that a single physical mechanism determines the exchange bias. There is overwhelming evidence that the origin of EB resides in the pinned, uncompensated moments (PUM) present in the AFM. The only possible exceptions are interfaces with sizeable Dzyaloshinskii-Moriya interaction [14], which breaks mirror symmetry and may lead to EB at perfectly compensated interfaces. Moreover, it is crucial that in addition to the PUM there is evidence for the presence of unpinned, uncompensated moments (UUM), which do not influence the EB, but may affect the coercivity [15]. It should also be mentioned that the presence of intentionally introduced non-magnetic sites (impurities and/or defects) may affect the domain state (and consequently the PUM) of the AFM as theoretically implied by the 
"Imry-Ma argument" [16]. It refers to the statistical imbalance of the number of impurities on the two AFM sublattices within any finite region, leading to a net AFM magnetization.

The following issues arise naturally regarding the PUM: a) Do they reside on the surface and/or in the bulk of the AFM? b) What is their microscopic origin? and c) do bulk spins/moments play any role? Generally it is assumed that EB is a purely interfacial effect in which bulk moments provide the pinning matrix for the interfacial PUM. However, recent experiments show clear evidence that bulk AFM spins/moments play an active role in determining the EB features.

In this "Critical Focused Issue" we will discuss this particular important characteristic and highlight unanswered questions that are still open for further research. We will not discuss the role of PUM at the AFM/FM interface as this has been extensively done in previous articles [17-20]. We will focus on pure type 1 and type 1-like AFM systems, in which thermal fluctuations play a minor role due to the high anisotropy, epitaxial nature, large grain size, relevant inter-gain coupling and/or low enough measurement temperature. This also excludes systems in which training and other history dependent phenomena may be associated with metastable magnetic structures, e.g. spin glasses,

present in the bulk of the AFM [21]. On the other hand, we exclude pure type 2 materials consisting of small, uncoupled AF grains as, e.g., IrMn. Their behavior has been addressed by a phenomenological model based on thermal activation of AFM grains with distributed grain sizes to explain loop shifts, training and changes in coercivity with temperature [22]. In this particular case the microscopic mechanism for the EB and the key role that PUM play is still elusive.

\section{EXPERIMENTAL EVIDENCE}

In this section, we will summarize the different classes of experiments which imply that bulk PUM play a major role in Exchange Bias. 


\subsection{Dilution in the bulk}

Uncompensated moments were generated intentionally only in the bulk AFM using nonmagnetic defects [23] and keeping the interface the same for all dilutions. Nonmagnetic defects create a statistical imbalance in the ideally equal number of spins in the two sublattices of the AFM. This imbalance results in a net number of uncompensated spins which couple to the external magnetic cooling field. This was accomplished in the strong-anisotropy AFM $\mathrm{CoO}$ by diluting the bulk magnetic Co sites with nonmagnetic $\mathrm{Mg}$ [23]. The samples were prepared from a ferromagnetic Co layer, grown on (0001)oriented single crystalline sapphire $\mathrm{Al}_{2} \mathrm{O}_{3}$ (Fig. 1(a)). To assure that all samples had an identical interface, a $0.4-\mathrm{nm}$ thick antiferromagnetic $\mathrm{CoO}$ layer containing a nominally minimum defect concentration was then deposited on top of the Co layer. The subsequently deposited epitaxial antiferromagnetic $\mathrm{CoO}$ layers were diluted by inserting nonmagnetic $\mathrm{Mg}$ substitutions in $\mathrm{Co}_{1-\mathrm{x}} \mathrm{Mg}_{\mathrm{x}} \mathrm{O}$ or Co defects in $\mathrm{Co}_{1-\mathrm{y}} \mathrm{O}$. In this fashion, a variable concentration of defects was generated away from the FM/AFM interface, within the volume part of

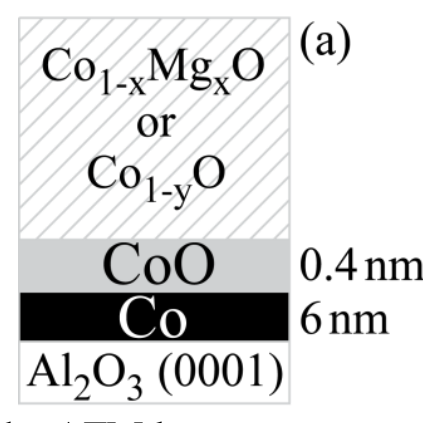

the AFM layer.

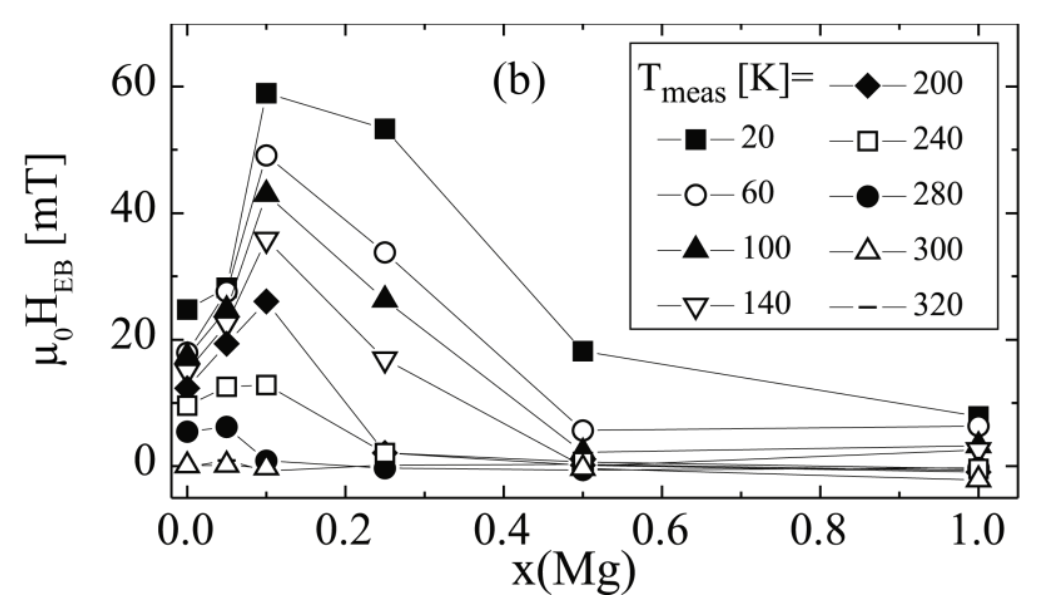

Fig. 1. (a) Experimental layer sequence and (b) enhancement of the EB field due to nonmagnetic $\mathrm{Mg}$ substitutions in the bulk region of the antiferromagnetic $\mathrm{CoO}$ as a function of temperature [23]. 
Fig. 1(b) shows the dependence of the $\mathrm{EB}$ as a function of $\mathrm{Mg}$ dilution $x$ in the $\mathrm{CoO}$ bulk at different temperatures. The changes in the bulk of the AFM cause major changes of the EB field. For example at $20 \mathrm{~K}$ the EB field is enhanced (over the background of about $20 \mathrm{mT}$ ) by a factor of three due to $10 \%$ nonmagnetic $\mathrm{Mg}$ dilution of $\mathrm{CoO}$, starting at a distance of $0.4 \mathrm{~nm}$ away from the $\mathrm{Co} / \mathrm{CoO}$ interface (see Fig. 1(a)).

This provides a strong proof that PUM in the bulk of the AFM (not solely at the interface with the FM) is an essential ingredient in EB. As a consequence, volume domains form in the AFM as the domain walls pass through these nonmagnetic defects with a minimum energetic cost. Hence the creation of PUM in the AFM bulk is intimately related to the formation of volume domains in the AFM. The model described in section 4 supports these domains and shows that the defect-induced domain state in the AFM bulk produces a small net remanent magnetization in the AFM bulk. This triggers the spin arrangement at the FM/AFM interface, which results in EB.

Similar results were reported for the diluted $A F M ~ F e F 2$ in $\mathrm{Fe}_{\mathrm{x}} \mathrm{Zn}_{1-\mathrm{x}} \mathrm{F}_{2} / \mathrm{Co}$ bilayers grown on $\mathrm{MgO}(100)$ [24]. Compared to the undiluted sample a $65 \%$ enhancement of the EB field was obtained by diluting the Fe by $17 \% \mathrm{Zn}$ and by inserting a 1.0-nm thick layer of pure $\mathrm{FeF}_{2}$ between the diluted AFM and the Co layer. The intervening pure AFM layer is found to increase the coupling between the FM and the diluted AFM layer.

\subsection{Radiation Damage}

A direct measurement of the effect of defects in the AFM bulk was investigated using He radiation damage of specially prepared $\mathrm{EB} \mathrm{Ni} / \mathrm{FeF}_{2}$ samples. The change in the defect density as a function of distance from the interface was controlled using a variable thickness $\mathrm{Au}$ wedge which determines the penetration depth of the He-ions. In this fashion, defects were produced in the AFM at different depths (Fig. 2). These experiments show that even when the defects produced by the He radiation are far from the FM/AFM interface, the exchange bias is substantially modified. This result can only 
be explained if the AFM bulk plays a role in the exchange bias through the production of PUM in the bulk.
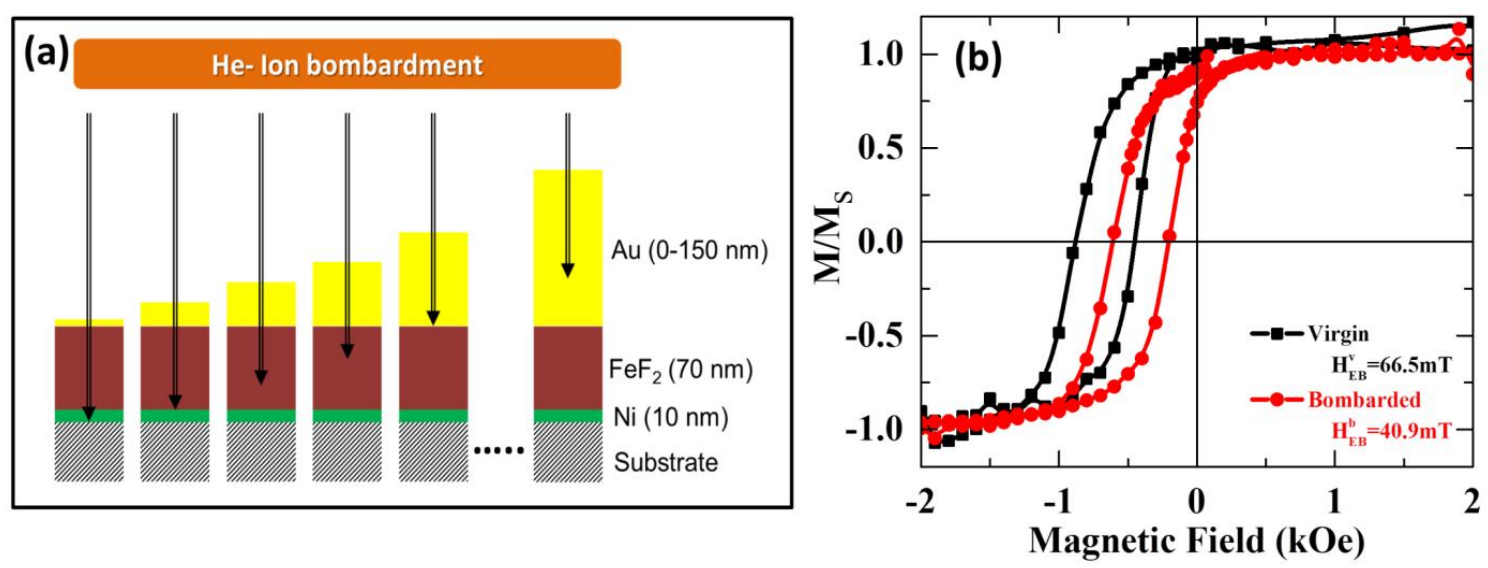

Fig. 2 (a) Sketch of the experimental approach. $\mathrm{Ni}(10 \mathrm{~nm}) / \mathrm{FeF}_{2}(70 \mathrm{~nm}) / \mathrm{Au}(\mathrm{t})$ multilayer films were grown in -situ with varying Au capping layer thickness. Heion bombardment yields defects at different depth, which is controlled by $\mathrm{Au}$ thickness. (b) Magnetization measurements for bilayer before (black squares) and after (red circles) He-ion bombardment for $30 \mathrm{~nm}$ of Au capping layer sample. The ions create defects only in the AFM bulk. Both curves are obtained at $10 \mathrm{~K}$ after 200 Oe field cooling from $200 \mathrm{~K}$. He-ion bombardment decreases the loop shift by $\sim 38 \%$. Magnetization values are normalized to saturation. Adapted from Ref. [25].

As a function of Au thickness a non-monotonic dependence of the EB was observed, indicating that the bulk of the AFM affects the results. Note that radiation damage had an effect even in those cases when only the bulk of the AFM was affected by the radiation. A maximum change of EB is observed if defects are created throughout the bulk of AFM layer and at the FM/AFM interface. This can be attributed to a balance of several mechanisms for which both bulk and interface need to be considered. The importance of the AFM bulk for determining EB is independent of these complex scenarios. 


\subsection{Multilayer}

FM1/AFM/FM2 multilayers with dissimilar ferromagnets were designed to investigate the role of bulk AFM spins. A direct evidence of an internal spin structure in the AFM coupling both FMs was demonstrated in $\mathrm{FeNi}(50 \mathrm{~nm}) / \mathrm{FeF}_{2}\left(t_{\mathrm{AFM}}\right) / \mathrm{Ni}(50 \mathrm{~nm})$ trilayers [26]. The different coercivity of Permalloy $\left(\mathrm{FeNi}\right.$ ) and $\mathrm{Ni}$ above the $\mathrm{FeF}_{2}$ Néel temperature allows field-cooling configurations with either parallel or antiparallel magnetization alignments of the FM layers (Fig. 3(a)). Magneto-optical Kerr effect was used to probe separately the magnetization reversal of each FM layer. Fig. 3(b) shows the EB as a function of temperature for the two FMs after parallel (solid symbols) and antiparallel (open symbols) field-cooling. Both interfaces are affected by the cooling configuration, Ni $H_{\mathrm{EB}}$ decreases one order of magnitude while $\mathrm{Py} H_{\mathrm{EB}}$ is reduced to half from the parallel to the antiparallel alignment. The large difference between parallel and antiparallel cooling conditions underlines the important role of the internal AFM spin structure. This magnetic structure determines the spin configuration at each FM/AFM interface and finally the EB magnitude. In other systems, like epitaxial $\mathrm{Fe} / \mathrm{MnPd} / \mathrm{Fe} / \mathrm{IrMn}$ multilayers, the parallel and antiparallel cooling procedure revealed a spin-flop transition and an internal AFM spin reorientation [27].



Fig. 3. (a) Cooling configurations. (b) Dependence of the Ni and Py exchange bias field of $\mathrm{Ni}(50 \mathrm{~nm}) / \mathrm{FeF}_{2}(200 \mathrm{~nm}) / \mathrm{Py}(50 \mathrm{~nm})$ trilayers for both parallel (solid symbols) and antiparallel (open symbols) cooling configurations. Adapted from Ref. [26]. 
Similar conclusions are drawn from a recent experiment with $\mathrm{Ni} / \mathrm{NiMn} / \mathrm{Ni}$ trilayers which combine in-plane and out-of-plane anisotropies [28]. Fig. 4(a) illustrates the fabrication steps. Magneto-optical measurements were performed in situ at each step, without breaking vacuum. Fig 4(b) shows the temperature dependence of the exchange bias field for the same ferromagnetic layer (bottom Ni, FM1) at two different fabrication steps. The EB of FM1 (always in-plane) is altered by switching the FM2 magnetization from out-of-plane to in-plane. Red circles correspond to the exchange bias of FM1 as the FM2 magnetization points out-of-plane. An ultrathin Co layer tilts the FM2 magnetization from out-of-plane to in-plane, and as a consequence the EB of FM1 is substantially reduced (blue triangles). This reduction is due to the presence of PUM in the AFM bulk, which magnetically connect both FM layers through exchange paths in the AFM.

(a)
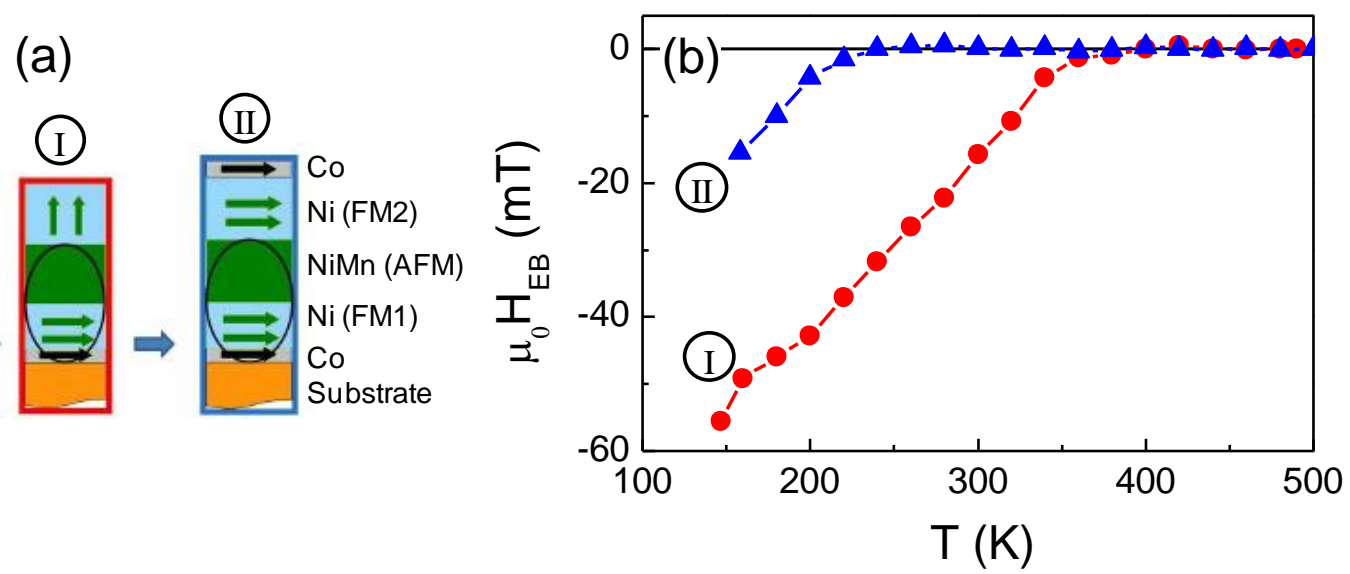

Fig. 4. (a) Fabrication steps of FM1/AFM/FM2 trilayers with in-plane/out of plane (I) and in-plane/in plane (II) configurations. (b) Temperature dependence of the exchange bias field of the bottom Ni layer, FM1, at two fabrication steps. Adapted with permission from Ref. [28].

Further evidence regarding the role of PUM in the AFM bulk was obtained by comparing the EB magnitude of FM1/AFM, AFM/FM2 bilayers with that of FM1/AFM/FM2 trilayers. Thus, symmetric $\mathrm{CoFe} / \mathrm{FeMn}\left(t_{\mathrm{AFM}}\right) / \mathrm{CoFe}$ trilayers show an enhanced $\mathrm{EB}$ in both FM layers for a smaller AFM thickness than in the bilayers. Moreover, equivalent $\mathrm{CoFe} / \mathrm{FeMn}$ interfaces exhibit larger EB in trilayers than in bilayers [29]. A similar result 
was found in FeNi/FeMn, FeMn/Co bilayers and FeNi/FeMn/Co trilayers [30]. The differences in the exchange bias field, for equivalent FM/AFM interfaces in bilayers and trilayers, can only be explained considering the bulk AFM spin structure.

\subsection{Artificial Bulk PUM}

The role of bulk PUM may be enhanced in artificial nanostructures. To do this, square arrays of $200 \mathrm{~nm}$ square antidots were patterned by focused ion beam (FIB) lithography through the whole depth of $\mathrm{Ni} / \mathrm{FeF}_{2}$ heterostructures (Figure 5(a)) [31]. The nominal antidot density (AD) was obtained as the ratio between the area of the patterned region and the total area of the sample, for $\mathrm{AD}=0.07,0.12$ and 0.24 , corresponding to side-toside distances of $683 \pm 12,373 \pm 10$, and $207 \pm 12 \mathrm{~nm}$, respectively.

The cooling field $\left(H_{\mathrm{FC}}\right)$ necessary to produce positive exchange bias (PEB) decreases as the $\mathrm{AD}$ density increases (Figure 5(b)) [32,33]. For example, for $\mathrm{AD}=0.24$, PEB appears at $H_{\mathrm{FC}}$ one order of magnitude smaller than for the unpatterned film, suggesting that the antidot patterning produces additional PUM [32,33]. The latter can be understood by taking into account the role of the artificially generated PUM throughout the AFM slab. Figure 5(a) illustrates the spin configuration yielding full PEB. Only PUM in the AFM are colored (compensated spins are not depicted). Blue arrows indicate PUM coupled to FM moments (gray arrows) at the interface. Red arrows correspond to PUM not coupled to the FM since they are not in direct contact. These "red-colored" spins are

artificially created during the patterning process throughout the carved AFM faces and correspond to non-interfacial (i.e. away from the FM-AFM interface) moments. All PUM (blue and red) depicted in Figure 5(a) belong to the same EB domain-volume in the AFM. Therefore all of them must be oriented below $T_{\mathrm{N}}$ during $H_{\mathrm{FC}}$ either antiparallel to the FM magnetization (yielding NEB) or parallel to the FM spins (yielding PEB) as in Figure 5(a).

The spin configuration at both sides of the FM/AFM interface was directly observed by photoemission electron microscopy (PEEM) combined with X-ray magnetic circular 
dichroism (XMCD). Besides, PEEM in this case is sensitive to the additional PUM present on the side-walls of the antidots. These measurements proved that in antidot containing samples additional PEB domains appear, when the Zeeman energy of bulk PUM locally overcome the exchange energy at the FM/AFM interface [34]. Independent PEEM experiments also suggested the presence of additional bulk PUM on the artificially-created, exposed lateral AFM walls as a result of the antidot carving [34]. Note also that an increase in inverted Ni domains is only observed below a certain antidot separation. Therefore, strain associated with patterning does not play a major role.

(a)

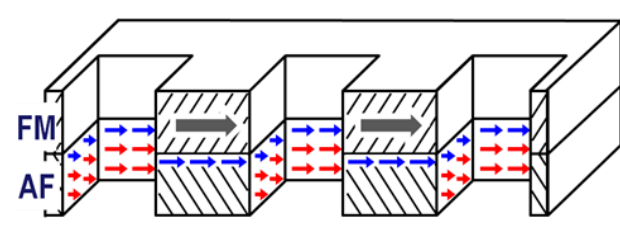

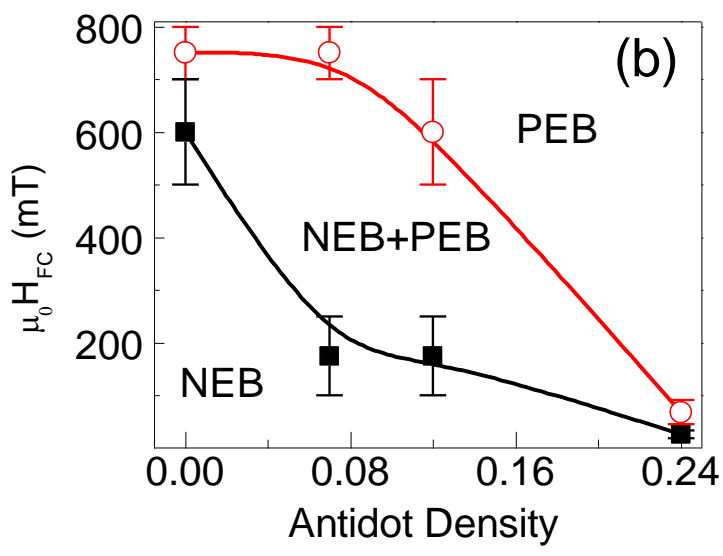

Fig. 5. (a) Cross section of $\mathrm{Ni} / \mathrm{FeF}_{2}$ antidots, showing the spin configuration of pinned uncompensated AFM moments for a PEB domain. "Blue" arrows represent AFM spins at the AFM/FM interface. "Red" arrows indicate bare AFM spins at exposed AFM faces. Wide black arrows indicate the FM magnetization direction. (b) Cooling fields, $\mu_{0} H_{\mathrm{FC}}$, corresponding to the onset of positive EB (PEB) (solid squares) and to those at which negative EB (NEB) disappears (empty circles), as function of the antidot density in $\mathrm{Ni} / \mathrm{FeF}_{2}$ bilayers. Dashed lines are guides to the eyes showing the region where the bidomain state takes place.

\section{4.- MODELS}

Several models have been developed in the past to account for effects related to the EB phenomenon. Many of these models make different assumptions regarding the nature of 
the pinned, uncompensated moments (PUM) that give rise to EB and some of them have never been tested by numerical simulations. To achieve EB there must be some interface magnetization in the AFM which couples to the FM. A purely interfacial moment maybe expected from atomically flat interfaces with geometrically (perhaps roughness induced) uncompensated moments. However, roughness tends to compensate the moments at these surfaces. For compensated AFM interfaces an AFM interface magnetization can only appear: i) statistically, when the AFM is of nanometer size or in a granular film [22], ii) when the collinear spin structure is distorted at the interface, iii) when the inversion symmetry of the crystal is broken, and a sizeable Dzyaloshinskii-Moriya interaction arises [14], or iv) when the long-range antiferromagnetic order in the bulk of the AFM is broken by the appearance of a domain structure in the AFM. The latter is the most general case and most realistic if imperfections are considered. The first model following this idea [35-37] implies that interface roughness produces domains in the AFM which will lead to PUM at the interface. However, the stability of these domains was not tested previously.

Another possible explanation for exchange bias in ,e.g., $\mathrm{FeF}_{2}$-based heterostructures, maybe considered attractive as an explanation of a limited set of experiments. Piezomagnetism has been reported for antiferromagnets with rutile structure such as $\mathrm{FeF}_{2}$ and piezomagnetic coupling in $\mathrm{FeF}_{2}$-based heterostructures was shown to change under external shear stress [38]. However, the lack of macroscopic evidence such as vertical shifts in the hysteresis loops, steep increase of the magnetization on cooling below $T_{\mathrm{N}}$ [38] and references therein, and/or a sizeable temperature dependent offset of the magnetization [39] in exchange biased bilayers imply that piezomagnetism doesn't play a major role even for $\mathrm{FeF}_{2}$ systems. On the other hand, the explanation outlined below has a universal appeal for all materials systems and geometries investigated to date.

The occurrence and stability of bulk domains in the AFM was investigated using a model that includes natural or intentionally introduced defects in the AFM bulk - the "domain state (DS)" model [23,40]. This model is classical, assuming local spins that describe a bilayer consisting of an FM layer coupled by nearest neighbor exchange to an AFM 
layer. The AFM contains some nonmagnetic spin sites as produced for instance by nonmagnetic impurities. This model was extensively studied numerically, mostly using Monte Carlo simulations, for Ising as well as Heisenberg spins [41,42]. Many of the experimental findings could successfully be described $[40,43]$ as shown for instance in a comparison between Fig. 6 and Fig. 1(b).

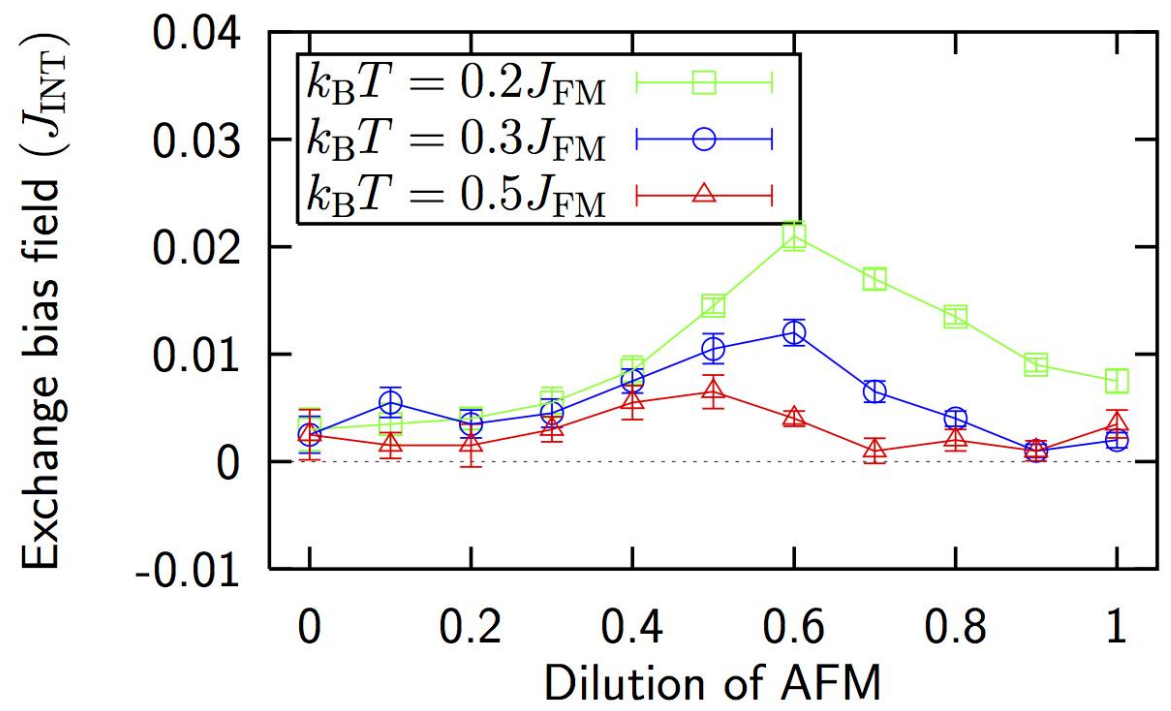

Fig. 6. EB field as a function of $\mathrm{Mg}$ concentration, $x$, from the domain state model applied to $\mathrm{Co} / \mathrm{Co}_{1-\mathrm{x}} \mathrm{Mg}_{\mathrm{x}} \mathrm{O}$. The dilution enhances the EB field by as much as $4-5 \%$ of the interface exchange coupling $J_{\text {INT }}[23]$.

The reason for the domain formation in the AFM is twofold; the interface coupling to the ferromagnet and the coupling of the AFM bulk to the externally applied cooling field. The exchange field stemming from the FM may point parallel or antiparallel to the external field. The AFM domains carry a net magnetization lowering the energy of the system (see Fig. 7, lighter grey region). This net magnetization arises from two contributions; the AFM domain walls (domain wall magnetization) and from an imbalance in the number of defects of the two AFM sub-lattices within a domain (volume magnetization). The reason for this imbalance is that the domain structure is not random. Rather, it is an optimized structure which minimizes the energy during the initial cooling procedure. As a consequence, the AFM magnetization which couples to the FM exchange field along the interface and to the external field is maximized. The AFM domain state 
magnetization produces an interface contribution which provides an exchange field for the FM in addition to the external field. The bulk thus stabilizes the domain structure against thermal fluctuations and against reversal of the FM during hysteresis.

These arguments for the domain formation are in line with the more general theoretical considerations that the ordered state of an infinite spin system can be unstable against random defects due to impurities or due to random fields. The original, so-called "ImryMa argument" [16], was developed for the random field Ising model. It is based on the energetics of domain formation following the fluctuations of the Zeeman energy in a random field. Later on it was established that a magnetically diluted AFM in an external magnetic field is in the same universality class [44-46] and develops a similar domain state when cooled below its Néel temperature [47,48].



Fig. 7. Sketch of a FM monolayer (yellow) in contact with a geometrically compensated AFM interface layer. Defects in the AFM are marked red. Due to these 
defects it is energetically favorable to reverse a region of the AFM bulk (lighter grey) to form a domain in the otherwise ordered AFM. The domain provides an interface field to the FM, leading to EB. Upon reversal of the FM the domain walls in the AFM domain will be pinned by defects at the interface and in the bulk of the AFM (red spheres). This pinning and the external cooling field stabilize the domain and its interface magnetization and lead to EB. The coercivity is enlarged by reversible changes of the domain structure, e.g., minor rearrangements of the domain wall structure between pinning centers.

Furthermore, simulations [40] showed that an AFM interface magnetization alone does not necessarily lead to EB. Only the pinned magnetization can lead to EB [15]. Following the dynamics of the spin configuration during the simulations it was shown that the major part of the AFM domain structure did not change during FM reversal. However, there are rearrangements on smaller length scales because the domain wall magnetization changes its sign at the interface while the volume magnetization arising from the defects remains frozen. The stability of the domain structure arises from the bulk of the AFM. Since the domain walls are pinned at defect sites and between pairs of spins which are aligned with the field, the motion of a domain wall must overcome energy barriers by thermal activation. This explains why a large domain in general will remain in a metastable state on exponentially-long time scales, while rearrangements on a shorter length scale (training effect) are possible, of course depending on the waiting time, temperature, and AFM material parameters.

The DS model correctly predicted that some dilution of the AFM bulk — which is conducive to formation of AFM domains - leads to an increased EB. The original calculations predicted an optimal dilution of the order of $60 \%$ (Fig. 6), while experiments found it to be of the order of 10\% (Fig. 1 (b)) [23]. Later modifications of the DS model, to include interface roughness, lead to a new estimate of the optimal dilution, much closer to experimental values and produced new insight regarding the dependence of EB on the details of the interface structure [49]. The model consists of an Ising AFM of thickness $t_{\mathrm{AFM}}$, coupled to a monolayer Heisenberg FM. A finite interface roughness was modeled by a single interface layer where FM spins were mixed with a certain probability $\mathrm{R}$ into 
the AFM. The reason for the enhanced EB lies in the optimized interface coupling associated with low values of R. EB is then greater in slightly rougher systems in accordance with the work of Malozemoff because it effectively enhances the FM-AFM coupling. The largest EB field is achieved for a rather small amount of FM spins in the mixed interface layer.

\subsection{Summary of the model}

The final picture which arises is quite simple and is illustrated in Fig. 8. Due to the domain structure in the AFM the interface layer is effectively uncompensated. The domain structure is stabilized in the bulk by rather sparse ( 5\%) PUM, located on the AFM lattice. The coupling between the PUM is provided by the AFM background. Because of this, the ordering temperature is related to the AFM Néel temperature, but the total uncompensated moment is quite small as compared to the total number of spins in the AFM. From all the above mentioned evidence it is clear that for AFM/FM heterostructures the presence of PUM in the bulk of the AFM plays a major role in the EB. PUM provide the necessary stability for the AFM domain structure which explains not only the occurrence of EB but also its temperature [40] and thickness dependence , as well as the training effect [50], among many other related effects.

The above description of the DS model is qualitatively summarized below by the schematic illustration (Fig. 8). The red dots denote defects (nonmagnetic ions or vacancies) and the solid line surrounds a domain in which the staggered magnetization is reversed with respect to the background staggered magnetization outside this domain. Note that the compensated AFM moments contribute on average zero magnetization, but provide the coupling between the uncompensated moments. For this particular case, the number of uncompensated moments (green arrows with circles) of the domain is three and the number of broken (frustrated) magnetic bonds (black crosses) at the domain boundary is four. Therefore, for $\mu \cdot \mu_{\mathrm{o}} H_{\mathrm{FC}}>4 / 3\left|\mathrm{~J}_{\mathrm{AFM}}\right|$ the shown moment configuration is stabilized by the cooling field $\mu_{\mathrm{o}} H_{\mathrm{FC}}$. 


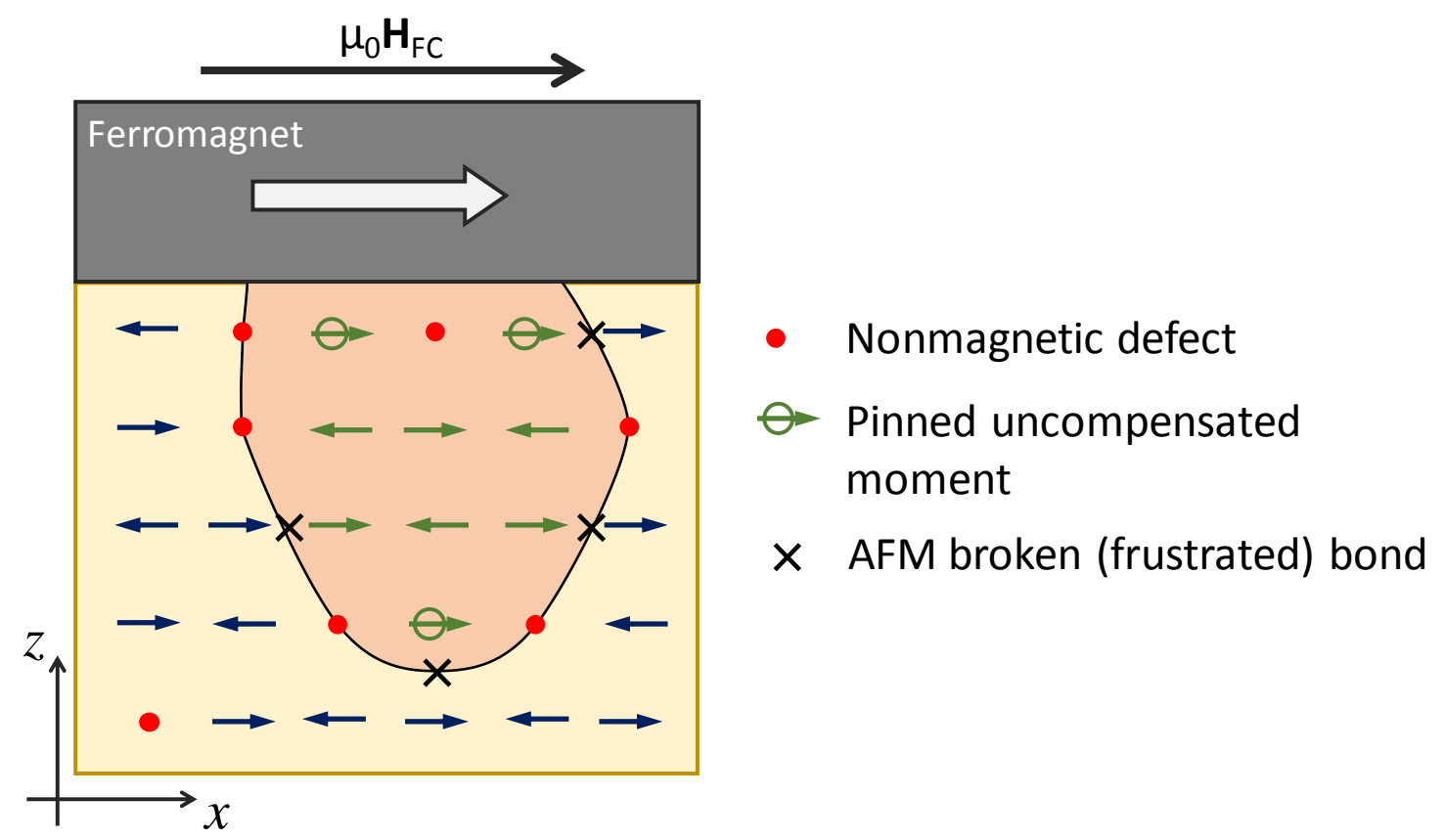

Fig. 8. Schematic illustration of a magnetic domain (enclosed by the solid line contour) in an AFM containing defects (nonmagnetic ions or vacancies -red dots), frustrated bonds between moments (marked by crosses X), and pinned uncompensated moments (green circled arrows) [51].

A quantitative correlation between the formation of a DS in an AFM or its bulk magnetization and the EB field was given for Co clusters in a disordered MnPt thin film [52]. It was shown that the effective field acting on the interface AFM magnetization, responsible for $\mathrm{EB}$, is proportional to the bulk magnetization. The authors consider this evidence as the true signature of the DS model. The cooling field aligns the uncompensated moments in the AFM. These field aligned bulk moments we consider as a new type of "ferromagnet". This represents a novel type of diluted, random, saturated ferromagnet, which interacts with the FM layer to yield EB. The AFM provides the spin background which couples the randomly located uncompensated spins. So this ferromagnet has the following properties: low moment given by the low uncompensated AFM moment density, a Curie temperature given by the AFM Néel temperature, and randomly distributed moments which, however, are located on the regular AFM lattice sites. 
Important issues remain that may be crucial for further improvements of the EB in heterostructures. Among these are, e.g., can simple guidelines be established for the optimum number of uncompensated moments? What are the materials parameters which determine the pinning strength of the PUM? Are there any new microstructures which can enhance the EB? Can EB be set by other means than cooling in a magnetic field? Can the number of PUM (and therefore the EB) be manipulated by external means such as stress, light, and electric field? Can the net antiferromagnetic moment in the bulk and at the interface be measured separately with sufficient accuracy so as to address some of the open issues highlighted here?

\section{CONCLUSIONS}

Exchange bias systems were divided into two classes: type 1, highly ordered epitaxial or textured systems whose behavior is largely governed by spin configurations produced by the structure, and type 2, polycrystalline systems for which thermal fluctuations play a major role. We have presented experimental and theoretical evidence that exchange bias in type 1 ferromagnetic/antiferromagnetic heterostructures is in grand part affected by the behavior of pinned uncompensated moments in the bulk of the AFM. In addition, the totality of experimental and theoretical concepts are well described by the idea that the pinned uncompensated moments in the AFM responsible for the EB, act as a low moment ferromagnet coupled by the AFM background and whose moments occupy randomly regular AFM sites. A large number of unanswered issues are highlighted given this novel viewpoint.

\section{ACKNOWLEDGEMENTS}

This is a highly collaborative manuscript. The outline was conceived jointly, the data and models were extensively debated and the paper was written by multiple iterations between all the coauthors. The research at UCSD was supported by the Office of Basic Energy Science, U.S. Department of Energy, BES-DMS funded by the Department of Energy's Office of Basic Energy Science, DMR under grant DE FG02 87ER-45332. 
Support from the Spanish MINECO (Grants No. MAT2015-68772-P, FIS2013-45469 and CAS12/00241), Catalan DURSI (2014SGR220), European Union FEDER funds (Una manera de hacer Europa), the 7th European Union Framework Programme (FP7PEOPLE-2012-IRSES, Project No. 318901) and the University of Barcelona (UB) is also acknowledged.

We thank Dr. A. Basaran for Fig. 2, and Prof. W. Kuch for allowing us to modify Fig. 4. X.B. is indebted to Prof. A. Labarta, Dr. A. Fraile-Rodríguez and Dr. M. Kovylina from $\mathrm{UB}$ for their work in Ni/FeF2 antidots and PEEM experiments.

\section{REFERENCES}

[1] For an experimental review see for instance, J. Nogués and I. K. Schuller, Exchange Bias, J. Magn. Magn. Mater. 192 (1999) 203.

[2] For a theoretical review see for instance, M. Kiwi, Exchange bias theory, J. Magn. Magn. Mater. 234 (2001) 584.

[3] W.H. Meiklejohn, Exchange Anisotropy - A Review, J. Appl. Phys. 33 (1962) 1328.

[4] K. Takano, R.H. Kodama, A.E. Berkowitz, W.. Cao, G. Thomas, Interfacial Uncompensated Antiferromagnetic Spins: Role in Unidirectional Anisotropy in Polycrystalline $\mathrm{Ni}_{81} \mathrm{Fe}_{19}$ / CoO Bilayers, Phys. Rev. Lett. 79 (1997) 1130.

[5] A.E. Berkowitz, K. Takano, Exchange anisotropy - a review, J. Magn. Magn. Mater. 200 (1999) 552. doi:10.1016/S0304-8853(99)00453-9.

[6] H. Ohldag, A. Scholl, F. Nolting, E. Arenholz, S. Maat, A.T. Young, et al., Correlation between Exchange Bias and Pinned Interfacial Spins, Phys. Rev. Lett. 91 (2003) 017203. doi:10.1103/PhysRevLett.91.017203.

[7] G. Vallejo-Fernandez, L.E. Fernandez-Outon, K. O'Grady, Antiferromagnetic grain volume effects in metallic polycrystalline exchange bias systems, J. Phys. DAppl. Phys. 41 (2008) 112001. doi:10.1088/0022-3727/41/11/112001.

[8] J. Sort, B. Dieny, J. Nogués, Exchange bias in antiferromagnetic-ferromagneticantiferromagnetic structures with out-of-plane magnetization, Phys. Rev. B. 72 (2005) 104412. doi:10.1103/PhysRevB.72.104412.

[9] J. Nogues, D. Lederman, T.J. Moran, I.K. Schuller, Positive Exchange Bias in $\mathrm{FeF}_{2}-\mathrm{Fe}$ Bilayers, Phys. Rev. Lett. 76 (1996) 4624.

[10] T. Gredig, I.N. Krivorotov, P. Eames, E.D. Dahlberg, Unidirectional coercivity enhancement in exchange-biased Co/CoO, Appl. Phys. Lett. 81 (2002) 1270. doi:10.1063/1.1498505.

[11] A. Hoffmann, Symmetry Driven Irreversibilities at Ferromagnetic- 
Antiferromagnetic Interfaces, Phys. Rev. Lett. 93 (2004) 097203.

doi:10.1103/PhysRevLett.93.097203.

[12] S. Brems, D. Buntinx, K. Temst, C. Van Haesendonck, F. Radu, H. Zabel, Reversing the Training Effect in Exchange Biased CoO / Co Bilayers, Phys. Rev. Lett. 95 (2005) 157202. doi:10.1103/PhysRevLett.95.157202.

[13] C. Binek, Training of the exchange-bias effect: A simple analytic approach, Phys. Rev. B. 70 (2004) 014421. doi:10.1103/PhysRevB.70.014421.

[14] R. Yanes, J. Jackson, L. Udvardi, L. Szunyogh, U. Nowak, Exchange Bias Driven by Dzyaloshinskii-Moriya Interactions, Phys. Rev. Lett. 111 (2013) 217202. doi:10.1103/PhysRevLett.111.217202.

[15] S. Roy, M.R. Fitzsimmons, S. Park, M. Dorn, O. Petracic, I. V. Roshchin, et al., Depth Profile of Uncompensated Spins in an Exchange Bias System, Phys. Rev. Lett. 95 (2005) 047201. doi:10.1103/PhysRevLett.95.047201.

[16] Y. Imry, S. Ma, Random-field instability of the ordered state of continuous symmetry, Phys. Rev. Lett. 35 (1975) 1399.

[17] M.R. Fitzsimmons, C. Leighton, A. Hoffmann, P.C. Yashar, J. Nogués, K. Liu, et al., Influence of interfacial disorder and temperature on magnetization reversal in exchange-coupled bilayers, Phys. Rev. B. 64 (2001) 104415. doi:10.1103/PhysRevB.64.104415.

[18] H. Ohldag, T.J. Regan, J. Stöhr, A. Scholl, F. Nolting, J. Lüning, et al., Spectroscopic Identification and Direct Imaging of Interfacial Magnetic Spins, Phys. Rev. Lett. 87 (2001) 247201. doi:10.1103/PhysRevLett.87.247201.

[19] C. Chien, V.S. Gornakov, V.I. Nikitenko, A.J. Shapiro, R.D. Shull, Hybrid domain walls and antiferromagnetic domains in exchange-coupled ferromagnet/antiferromagnet bilayers, Phys. Rev. B. 68 (2003) 014418. doi:10.1103/PhysRevB.68.014418.

[20] Y. Fan, K.J. Smith, G. Lüpke, A.T. Hanbicki, R. Goswami, C.H. Li, et al., Exchange bias of the interface spin system at the Fe/MgO interface., Nat. Nanotech. 8 (2013) 438. doi:10.1038/nnano.2013.94.

[21] M. Ali, P. Adie, C.H. Marrows, D. Greig, B.J. Hickey, R.L. Stamps, Exchange bias using a spin glass., Nat. Mater. 6 (2007) 70. doi:10.1038/nmat1809.

[22] K. O’Grady, L.E. Fernandez-Outon, G. Vallejo-Fernandez, A new paradigm for exchange bias in polycrystalline thin films, J. Magn. Magn. Mater. 322 (2010) 883. doi:10.1016/j.jmmm.2009.12.011.

[23] P. Miltényi, M. Gierlings, J. Keller, B. Beschoten, G. Güntherodt, U. Nowak, et al., Diluted antiferromagnets in exchange bias: proof of the domain state model, Phys. Rev. Lett. 84 (2000) 4224..

[24] H. Shi, D. Lederman, E.E. Fullerton, Exchange bias in $\mathrm{Fe}_{\mathrm{x}} \mathrm{Zn}_{1-\mathrm{x}} \mathrm{F}_{2} / \mathrm{Co}$ bilayers, J. Appl. Phys. 91 (2002) 7763. doi:10.1063/1.1452266.

[25] A.C. Basaran, T. Saerbeck, J. de la Venta, H. Huckfeldt, A. Ehresmann, I.K. Schuller, Exchange bias: The antiferromagnetic bulk matters, Appl. Phys. Lett. 
105 (2014) 072403. doi:10.1063/1.4893457.

[26] R. Morales, Z.-P. Li, J. Olamit, K. Liu, J.M. Alameda, I.K. Schuller, Role of the Antiferromagnetic Bulk Spin Structure on Exchange Bias, Phys. Rev. Lett. 102 (2009) 097201. doi:10.1103/PhysRevLett.102.097201.

[27] W. Zhang, K.M. Krishnan, Spin-flop coupling and rearrangement of bulk antiferromagnetic spins in epitaxial exchange-biased Fe/MnPd/Fe/IrMn multilayers, Phys. Rev. B. 86 (2012) 054415. doi:10.1103/PhysRevB.86.054415.

[28] M.Y. Khan, C.-B. Wu, W. Kuch, Pinned magnetic moments in exchange bias: Role of the antiferromagnetic bulk spin structure, Phys. Rev. B. 89 (2014) 094427. doi:10.1103/PhysRevB.89.094427.

[29] D.N.H. Nam, W. Chen, K.G. West, D.M. Kirkwood, J. Lu, S.A. Wolf, Propagation of exchange bias in $\mathrm{CoFe} / \mathrm{FeMn} / \mathrm{CoFe}$ trilayers, Appl. Phys. Lett. 93 (2008) 152504. doi:10.1063/1.2999626.

[30] C.W. Leung, M.G. Blamire, Interaction between exchange-bias systems in $\mathrm{Ni}_{80} \mathrm{Fe}_{20} \mathrm{Fe}_{50} \mathrm{Mn}_{50}$ Co trilayers, Phys. Rev. B. 72 (2005) 054429. doi:10.1103/PhysRevB.72.054429.

[31] M. Kovylina, M. Erekhinsky, R. Morales, I.K. Schuller, A. Labarta, X. Batlle, The fabrication of ordered arrays of exchange biased $\mathrm{Ni} / \mathrm{FeF}_{2}$ nanostructures., Nanotechnology. 21 (2010) 175301. doi:10.1088/0957-4484/21/17/175301.

[32] M. Kovylina, M. Erekhinsky, R. Morales, J.E. Villegas, I.K. Schuller, A. Labarta, et al., Tuning exchange bias in $\mathrm{Ni} / \mathrm{FeF}_{2}$ heterostructures using antidot arrays, Appl. Phys. Lett. 95 (2009) 152507. doi:10.1063/1.3248306.

[33] R. Morales, M. Kovylina, I.K. Schuller, A. Labarta, X. Batlle, Antiferromagnetic/ferromagnetic nanostructures for multidigit storage units, Appl. Phys. Lett. 104 (2014) 032401. doi:10.1063/1.4862317.

[34] A. Fraile Rodríguez, A.C. Basaran, R. Morales, M. Kovylina, J. Llobet, X. Borrisé, et al., Manipulation of competing ferromagnetic and antiferromagnetic domains in exchange-biased nanostructures, Phys. Rev. B. 92 (2015) 174417. doi:10.1103/PhysRevB.92.174417.

[35] A.P. Malozemoff, Random-field model of exchange anisotropy at rough ferromagnetic-antiferromagnetic interfaces, Phys. Rev. B. 35 (1987) 3679.

[36] A.P. Malozemoff, Heisenberg-to-Ising crossover in a random-field model with uniaxial anisotropy, Phys. Rev. B. 37 (1988) 7673.

[37] A.P. Malozemoff, Mechanisms of exchange anisotropy (invited), J. Appl. Phys. 63 (1988) 3874. doi:10.1063/1.340591.

[38] C. Binek, X. Chen, A. Hochstrat, W. Kleemann, Exchange bias in $\mathrm{Fe}_{0.6} 6 \mathrm{Zn}_{0.4} \mathrm{~F}_{2}$ / Fe heterostructures, J. Magn. Magn. Mater. 240 (2002) 257.

[39] J. Nogués, T.. J. Moran, D. Lederman, I.K. Schuller, K. V. Rao, Role of interfacial structure on exchange-biased $\mathrm{FeF}_{2}-\mathrm{Fe}$, Phys. Rev. B. 59 (1999) 6984.

[40] U. Nowak, K.D. Usadel, J. Keller, P. Miltényi, B. Beschoten, G. Güntherodt, Domain state model for exchange bias. I. Theory, Phys. Rev. B. 66 (2002) 014430. 
doi:10.1103/PhysRevB.66.014430.

[41] U. Nowak, A. Misra, K.D. Usadel, Modeling exchange bias microscopically, J. Magn. Magn. Mater. 240 (2002) 243.

[42] A. Misra, U. Nowak, K.D. Usadel, Control of exchange bias by diluting the antiferromagnetic layer, J. Appl. Phys. 93 (2003) 6593. doi:10.1063/1.1543880.

[43] J. Keller, P. Miltényi, B. Beschoten, G. Güntherodt, U. Nowak, K.D. Usadel, Domain state model for exchange bias. II. Experiments, Phys. Rev. B. 66 (2002) 014431. doi:10.1103/PhysRevB.66.014431.

[44] S. Fishman, A. Aharony, Random field effects in disordered anisotropic antiferromagnets, J. Phys. C Solid St. Phys. 12 (1979) L729.

[45] J.L. Cardy, Random-field effects in site-disordered Ising antiferromagnets, Phys. Rev. B. 29 (1984) 505.

[46] A.K. Hartmann, U. Nowak, Universality in three dimensional random-field ground states, Eur. Phys. J. B. 7 (1999) 105.

[47] W. Kleemann, Random-field induced antiferromagnetic, ferroelectric and structural domains states, Int. J. Mod. Phys. B. 7 (1997) 2469.

[48] D.P. Belanger, Experiments on the random field Ising model, in Spin Glasses and Random Fields, World Scientific, Singapore, 1998.

[49] J. Spray, U. Nowak, Exchange bias in ferromagnetic/antiferromagnetic bilayers with imperfect interfaces, J. Phys. D-Appl. Phys. 39 (2006) 4536. doi:10.1088/0022-3727/39/21/003.

[50] A.G. Biternas, R.W. Chantrell, U. Nowak, Behavior of the antiferromagnetic layer during training in exchange-biased bilayers within the domain state model, Phys. Rev. B. 82 (2010) 134426. doi:10.1103/PhysRevB.82.134426.

[51] For clarity, and general purpose, Fig. 8 sketches a ferromagnetic coupling between AFM and FM moments at the interface, yielding conventional negative EB. For magnetic systems with antiferromagnetic coupling at the interface this configuration leads to positive EB at high cooling fields.

[52] R. Morel, C. Portemont, A. Brenac, L. Notin, Domain State and Exchange Coupling in MnPt with Co Clusters, Phys. Rev. Lett. 97 (2006) 127203. doi:10.1103/PhysRevLett.97.127203. 